\title{
SIRT1 in Secretory Organ Cancer
}

\author{
Raffaele Frazzi* \\ Laboratory of Translational Research, Azienda Unità Sanitaria Locale - IRCCS di Reggio Emilia, Reggio Emilia, Italy
}

Mammalian silent information regulator 1 (SIRT1) is reported to play a role in cancers of the secretory organs, including thyroid, pancreatic endocrine, and ovarian tumors (1-4). A recent meta-analysis conducted on 37 selected studies of human cancers analyzed the correlations of overall survival (OS), disease-free survival (DFS) and relapse-free survival (RFS) with SIRT1 expression (5). This study reported that SIRT1 overexpression was associated with a worse OS in liver and lung cancers, while it was not correlated with OS in breast cancer, colorectal cancer, or gastric carcinoma. Collectively, the meta-analysis revealed that an unfavorable OS was associated with SIRT1 expression for solid malignancies. Given the growing importance of this class of lysine/histone deacetylases in human endocrine malignancies, a rational and focused literature assessment is desirable in light of future clinical translations.

\section{OPEN ACCESS}

Edited by:

Yang Yang,

Northwest University, China

Reviewed by:

Zhenxing Liang,

First Affiliated Hospital of Zhengzhou

University, China

Daniel Christian Hoessli,

University of Karachi, Pakistan

Shashwat Sharad,

Center for Prostate Disease Research

(CPDR), United States

*Correspondence:

Raffaele Frazzi

raffaele.frazzi@ausl.re.it

Specialty section:

This article was submitted to

Cellular Endocrinology,

a section of the journal

Frontiers in Endocrinology

Received: 10 May 2018 Accepted: 05 September 2018 Published: 24 September 2018

Citation:

Frazzi R (2018) SIRT1 in Secretory

Organ Cancer.

Front. Endocrinol. 9:569.

doi: 10.3389/fendo.2018.00569
Keywords: SIRT1, cancer, secretory organs, acetylation, epigenetic modulation

\section{INTRODUCTION}

The epigenetic regulation of chromatin structure and gene expression represents a major field of study and intervention for researchers focused on cancer. The class III lysine/histone deacetylases are known as Sirtuins and are represented, in humans, by 7 members $(6,7)$. Silent information regulator 1 (SIRT1) is the most studied and well characterized among the class III deacetylases and its targets include p53, Ku70, peroxisome proliferator-activated receptor gamma (PPAR $\gamma$ ), peroxisome proliferator-activated receptor gamma coactivator 1-alpha (PGC1 $\alpha$ ), Beclin 1 and $\beta$-catenin, among others (8-13). A relevant group of SIRT1 targets is the Forkhead box O (FOXO) family of transcription factors. FOXO3a hyperacetylation mediated by SIRT1 accompanies apoptosis while FOXO4 deacetylation activates this target enhancing its transcriptional and biological activity in the nucleus. FOXO4 activation enhances the cellular defenses against oxidative stress and leads to apoptosis resistance $(8,14,15)$. Fibrosis is another aging-related disease involving FOXO1/3, well known modulators of aging and longevity playing a clear inhibitory effects on fibrogenic effector cells and extracellular matrix production (16).

Furthermore, the epigenetic regulation and dysregulation of the hypermethylated in cancer1 (HIC1)/SIRT1/p53 axis is relevant for the development of malignancies (17). SIRT1 is also a target for several miRNAs, small non-coding RNA molecules known to be deregulated in various cancers, whose expression is involved in tumorigenesis (18).

SIRT1, due to its $\mathrm{NAD}^{+}$dependency, is also a metabolic sensor and its deacetylating activity toward regulatory target proteins affects cell metabolism (19).

The focus of this review is the role and involvement of SIRT1 in secretory organ cancers since the information recently gathered on this topic should bear new translational benefits. Correlations between SIRT1 and nuclear receptors, transcription factors, master regulators of gene expression, miRNAs and lncRNAs have been reported. The main glands in the body and the most recent literature are taken into consideration. 


\section{OVARIAN CANCER}

Ovarian cancer is the fifth leading cause of female cancer mortality in the United States (20). This type of cancer is currently treated with a platinum and taxane-based chemotherapy after surgical cytoreduction. However, the rates of progression free survival (PFS) and OS for these patients remain dismal and no benefits seem to arise by the introduction of additional cytotoxic agents (21). Ovarian cancer is usually regarded as a single entity and current treatment protocols for the disease are not subtype specific. Actually, ovarian cancer is a heterogeneous disease that is classified by histopathological examination, including serous, clear cell, endometrioid, and mucinous subtypes. These subtypes develop differently and respond differently to chemotherapy (22).

SIRT1 overexpression is reported by many authors as associated with poor outcome and chemoresistance in ovarian cancer of epithelial origin (23-25). Ovarian cells (both normal and tumoral) express two kinds of estrogen receptors (ER), $\mathrm{ER} \alpha$ and $\mathrm{ER} \beta$ which play different roles in cell proliferation and aggressiveness (4). Specifically, ER $\alpha$ is associated to a poor outcome, while ER $\beta$ expression corresponds, on the contrary, to a favorable outcome. ER $\alpha$ levels are closely associated with estrogen-dependent growth and the increased metastatic potential of ovarian epithelial carcinoma (OEC) through the promotion of the epithelial to mesenchymal transition (EMT) (26). ER $\beta$, in contrast, inhibits EMT in presence of $17 \beta$-estradiol (E2), thus mediating the opposite effect to ER $\alpha$ (26). SIRT1 is inversely correlated to ER $\beta$ mRNA and protein levels, and the specific ER $\beta$ activator KB9520 strongly inhibits SIRT1 mRNA expression. These data collectively support the role of SIRT1 as a tumor promoter in OEC (4).

Recently, another association between OEC and SIRT1 was reported (27). These observations are relevant, because OEC patients often acquire resistance to paclitaxel or cisplatin, and the increased expression in SIRT1 and TWIST1 (two genes associated with drug resistance) is observed in OVCAR-5 cells that show increased cisplatin resistance, migration and invasion potential (27).

SIRT1 is a downstream target of hypoxia inducible factor $1 \alpha$ (HIFla) (28). Hypoxia is a condition associated with several types of tumors and, specifically, increased HIF1 $\alpha$ expression predicts the poor prognosis of ovarian cancer. Notably it has been demonstrated that SIRT1 expression is induced by hypoxia and that HIF1 $\alpha$ silencing indirectly hampers SIRT1 expression. Finally, the NF-кB signaling pathway is involved in hypoxiainduced SIRT1 up-regulation, strengthening the link between this class III lysine deacetylase and ovarian cancer (28).

\section{THYROID CANCER}

The incidence of thyroid cancer has increased over the past few decades worldwide. This increase is mainly driven by new cases of papillary thyroid cancer (29). SIRT1 is reported to be oncogenic in thyroid and prostate murine carcinomas initiated by PTEN deficiency (2). The mechanism unveiled by mRNA transcriptional analysis revealed that SIRT1 drives oncogenesis through c-MYC regulation (Figure 1). Two pathways upregulated via SIRT1 overexpression are related to translation and ribosomal biogenesis, which are both controlled by c-MYC. The protein product of the c-MYC oncogene lies at the intersection of a transcriptional network regulating cellular proliferation, replicative potential, cell-cell competition, cell size, differentiation, metabolism, and apoptosis (30).

c-MYC increases in response to mitogenic stimuli, and this is accompanied by SIRT1 upregulation. c-MYC activates SIRT1 by promoting NAMPT transcription and then the NAD-salvage pathway. Furthermore, c-MYC can bind directly to DBC1 (that is a SIRT1 inhibitor) thus preventing SIRT1 blockade $(31,32)$. On the other hand, SIRT1 activation leads to p53 deacetylation and consequent inactivation, decreasing the levels of PUMA and p21 and eventually counteracting the pro-apoptotic effects of p53. Finally, SIRT1 stabilizes c-MYC via lysine-specific deacetylation and increases its transcriptional activity. Altogether, these data demonstrate a positive feedback loop between c-MYC and SIRT1 (31).

The translational significance of these findings is highlighted by the consistent overexpression of SIRT1 in follicular and papillary thyroid carcinomas, positively correlates with c-MYC protein levels and stabilizes c-MYC (2).

The natural phytochemical resveratrol (RSV) is known to affect mammalian cell metabolism and aging by modulating SIRT1 $(33,34)$. Furthermore, SIRT1 is also a reported molecular target of RSV in cancer cells of both lymphoid and epithelial origin (35). RSV regulates thyroid stimulating hormone (TSH) secretion via SIRT1 activation. RSV also arrests follicular thyroid and papillary thyroid carcinoma growth by activating the mitogen-activated protein kinase (MAPK) signal transduction pathway and $\mathrm{p} 53$ phosphorylation $(36,37)$.

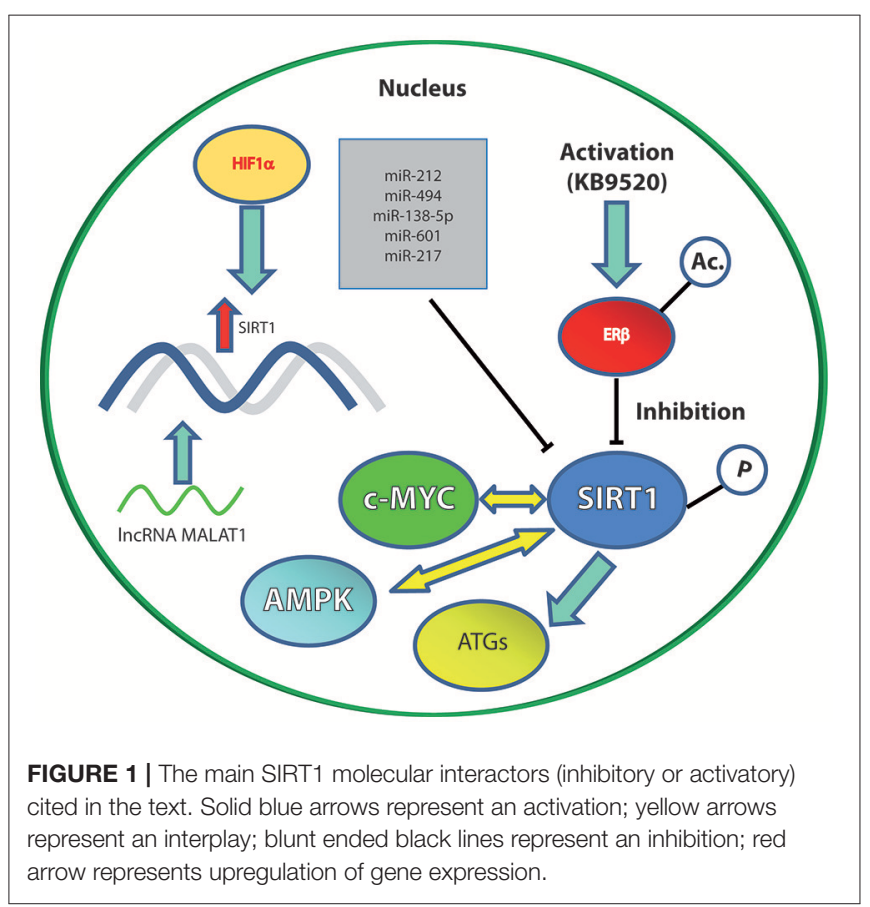


Among the few studies available, regarding the role of SIRT1 in the thyroid neoplasias, most report involvement as a direct target for miRNAs. miR-212 is a tumor suppressor miRNA that inhibits the proliferation, migration, and invasiveness of thyroid cancer cells. SIRT1 is a direct target of miR-212 and is inversely correlated to miR-212 expression in thyroid cancer tissues (42 samples of human thyroid cancer) (38).

\section{PANCREATIC CANCER}

Pancreatic cancer (PC) is one of the most deadly tumors in western countries and the 10th most common cancer in the United States. Pancreatic ductal adenocarcinoma is the most common subtype and is recognized as "incurable" due to its aggressive clinical course and resistance to available therapies (39, 40). miR-494 is specifically involved in PC proliferation (41). The overexpression of miR-494 inhibits PC cell proliferation in vitro and in vivo in addition to inhibiting invasion. The c-MYC/SIRT1 axis is a direct target of miR-494 and there is an inverse correlation between the two. The simultaneous interference of cMYC and SIRT1 synergistically reduces the expression of these two targets and inhibits PC proliferation, eventually stimulating miR-494 expression (41). The interplay between c-MYC and SIRT1, therefore, plays a relevant role during PC tumorigenesis and should be taken into account when designing strategies aimed at combating this cancer.

Recent studies on the miRNA-mediated regulation of autophagy in PC have been published (42). Since it is known that hypoxia induces autophagy, PC cells were cultured under normoxic and hypoxic conditions. miR-138-5p emerged among the most down-regulated miRNAs in the hypoxia-grown cells. Targets can analysis further revealed that the SIRT1 gene contains a classical and conserved domain that binds miR-138-5p. Notably, miR-138-5p specifically binds to the $3^{\prime}$-UTR of SIRT1 and its overexpression leads to SIRT1 protein downregulation (42). Finally, the authors demonstrated that miR-138-5p also counteracts the hypoxia induction in cancer cells through SIRT1 inhibition in tumor xenografts.

Another player that emerged recently is miR-601, which suppresses PC proliferation and migration (43). The target of this miRNA's activity was identified, once more, as SIRT1. This Sirtuin is downregulated by miR-601 and its overexpression reverses the effect of miR-601 on PC cells (43).

miR-217 also directly targets SIRT1, it is inversely correlated to SIRT1 expression and is down-regulated in chronic pancreatitis and PC (44).

The Ex-527 synthetic inhibitor, which inhibits pancreatic cell proliferation in vitro and in combination with the first-line drug for this pathology, gemcitabine, is considered one of the most specific SIRT1 inhibitors currently available (45). The same inhibitor was shown to promote PC xenograft tumors in SCID mice and did not synergize with gemcitabine. Therefore, caution should be used when dealing with Ex-527 inhibitor to target SIRT1 in vivo.

The role of histone deacetylases (HDACs) is poorly understood in pancreatic neuroendocrine tumors (pNETs).
A comprehensive expression pattern of all the five classes (I, IIa, IIb, III, and IV) of HDACs was recently described (3). The gene expression profiles of a total of $n=57$ patients revealed a significant upregulation of all the HDAC classes in pNETs over controls with increased levels ranging from 1.5- to 7-fold. The expression of several HDACs, including SIRT1, correlates to the G3 stage and, thus, to pNETs tumor grading (3).

\section{GASTRIC CANCER}

Gastric cancer (GC) is among the most prevalent malignancies of the upper gastrointestinal tract. It is a solid aggressive carcinoma where new therapeutic options are warranted (46). Autophagy in GC may have the dual role of promoting the activity of irradiation and anticancer drugs or, on the contrary, may function as a protective mechanism for cancer cells (47-49).

SIRT1 plays a confirmed role in autophagy $(50,51)$. In GC, it has been demonstrated that SIRT1 deacetylates autophagyrelated gene products (ATGs) and a series of histonic and non-histonic targets, eventually interfering with the autophagic process (Figure 1) (52).

GC is among the few human tumors where SIRT1 is downregulated, and in one study, the prognosis of lowSIRT1 expressing GC patients was good (52, 53). However, other authors reported a meta-analysis of SIRT1 expression in gastrointestinal cancer in which the results were heterogeneous (54). The overall results from 15 studies showed an association between worse OS and high SIRT1 expression in gastrointestinal cancer. The subgroup analysis revealed that this association was particularly strong in non-colorectal gastrointestinal cancers, especially GC and hepatocellular carcinoma (54). Therefore, SIRT1 is a promising prognostic factor in GC but not in colorectal tumors.

In contrast with the abovementioned results, a study of the clinicopathological parameters in GC patients was analyzed using the Kaplan-Meier plotter (55). Here, the data concerning the seven human Sirtuins were extrapolated from NCBI GEO databases containing mRNA profiles and the corresponding clinical data of a large number of GC patient samples. The Kaplan-Meier plotter was used to investigate the predictive value of mRNA expression of the Sirtuins for the OS of GC patients. Among a total of 631 available cases for SIRT1 expression, the survival curves suggested that a high expression of SIRT1 mRNA was favorable for OS and that different Sirtuins had diverse correlations with OS. Furthermore, the first progression (FP) was positively correlated with SIRT1 expression: in other words, a longer FP time was associated with a higher SIRT1 level (55).

Opposite evidence was reported from the immunohistochemical (IHC) staining of primary GC tissues. High SIRT1 expression, by IHC, was associated with lymphatic invasion $(p=0.028)$, vessel invasion $(p=0.016)$ and lymph node metastasis $(p=0.014)$ and tended to be associated with more advanced disease stages (56).

Clearly, no consistent data on the exact role of SIRT1 in GC are available at present. The evidence available thus far points to a dual role for SIRT1 (that is to say it may act as a tumor 
promoter as well as a tumor suppressor), possibly depending on its sub-cellular localization, p53 status, and microenvironmental conditions $(57,58)$.

\section{ADRENAL GLANDS}

The function of SIRT1 in the adrenal glands has been investigated the least. One study reports the researches performed on human adrenal NCI-H295R cells (59) and, in this setting, Sirtuins (including SIRT1) did not seem to be involved in the RSVmediated lowering of androgen production.

Moreover, steroidogenesis in H295R cells was not affected by Sirtuins, even when SIRT1/3/5 are overexpressed through transgenesis, strengthening the fact that RSV-dependent effects in this setting are SIRT-independent (59).

\section{HEPATIC CANCER}

Hepatocellular carcinoma (HCC) is a highly malignant tumor with a higher frequency in East Asia than in Europe and North America (60). HCC is the predominant malignancy in the liver and recent evidence shows a role for SIRT1/PGC1alpha/mitochondrial biosynthesis pathway in protecting the liver against lipid accumulation and mitochondrial dysfunction $(61,62)$. Senescence induction is a recently proposed strategy with the aim of counteracting HCC (63). Metformin, for instance, induce senescence via the activation of AMP-activated protein kinase (AMPK) when used at low doses. This effect is accompanied by the phosphorylation of SIRT1 as well as p53acetylation (Figure 1). The prolonged exposure to low doses of metformin leads to human HCC senescence in murine xenografts via modulation of the AMPK-SIRT1 axis (63).

The long non-coding RNA (lncRNA) metastasis associated lung adenocarcinoma transcript 1 (MALAT1) was recently discovered to interfere with SIRT1 activity in hepatocellular cancer (64). MALAT1 is frequently upregulated in several types of cancers and contributes functionally to the development and malignancy of tumor cells (65). MALAT1 is, among lncRNAs, a stable and highly transcribed molecule localized in the nucleus, and it has been reported that, in breast cancer cells expressing $\mathrm{ER} \alpha, 17 \beta$-estradiol treatment negatively regulates MALAT1 transcription, thus inhibiting proliferation, migration and invasion (66). MALAT1 is also regulated during endothelial differentiation in that hypoxia induces its transcription leading to the proliferation of the vasculature (67). In HCC specifically this lncRNA promotes aggressiveness in that releases SIRT1 by sponging miR-204 (64). SIRT1 is a direct downstream target of miR-204 and results inhibited by this interaction. MALAT1 can release the suppression on SIRT1 by base-pairing with miR-204 and leading to its post-transcriptional downregulation (64).

\section{CONCLUSIONS}

SIRT1 should be regarded as a multi-faceted enzyme and, as already suggested by several authors, studied in a contextdependent fashion. Secretory organs are no exception
TABLE 1 | Cancer models of the secretory organs in which SIRT1 has been studied.

\begin{tabular}{|c|c|c|}
\hline & $\begin{array}{l}\text { Experimental models and } \\
\text { specimens }\end{array}$ & References \\
\hline $\begin{array}{l}\text { Ovarian cancer of } \\
\text { epithelial origin }\end{array}$ & $\begin{array}{l}\text { Normal ovaries, endometriosis } \\
\text { with/without carcinoma, OvCa } \\
\text { (endometrioid; clear cell; mucinous; } \\
\text { serous). OvCa cell lines. Serous EOC. }\end{array}$ & $(4,23-28)$ \\
\hline Thyroid carcinoma & $\begin{array}{l}\text { Murine thyroid cancers. Thyroid cell } \\
\text { lines. Nude mice models. Human } \\
\text { normal and tumor thyroid specimens }\end{array}$ & $(2,37,38)$ \\
\hline Pancreatic cancer & $\begin{array}{l}\text { Confirmed human PC and non-tumor } \\
\text { pancreatic specimens. PC cell lines. } \\
\text { Normal human pancreatic ductal } \\
\text { epithelium. PC xenografts. Human } \\
\text { chronic pancreatitis specimens }\end{array}$ & $(41-45)$ \\
\hline $\begin{array}{l}\text { Pancreatic } \\
\text { neuroendocrine } \\
\text { tumors }\end{array}$ & $\begin{array}{l}\text { Formalin fixed paraffin-embedded } \\
\text { human samples }\end{array}$ & (3) \\
\hline Gastric cancer & $\begin{array}{l}\text { Human primary GC specimens. GC } \\
\text { cell lines. Clinical trials concerning } \\
\text { gastrointestinal cancers, overall } \\
\text { survival and SIRT1. } \\
\text { NCBI GEO databases of mRNA } \\
\text { profiles. Formalin fixed } \\
\text { paraffin-embedded human samples }\end{array}$ & $(52-56)$ \\
\hline Hepatic cancer & $\begin{array}{l}\text { Primary liver cancer organoids } \\
\text { (hepatocellular carcinoma; } \\
\text { cholangiocarcinoma; combined } \\
\text { HCC/CC; healthy liver). HCC mouse } \\
\text { xenografts. HCC cell lines and } \\
\text { primary human tissues }\end{array}$ & $(61,63,64)$ \\
\hline
\end{tabular}

to this. Table 1 summarizes the secretory organ tumors in which SIRT1 has been investigated, with the relevant experimental models. The most recent publications are taken into account.

SIRT1 plays a confirmed role in ovarian, thyroid, and pancreatic cancers, while its role in GC is yet to be definitely established. The evidence available in thyroid cancer confirms that c-MYC and SIRT1 are valuable therapeutic targets, encouraging translational researches aimed at the related pathways. The recent meta-analysis spanning 37 selected studies on human solid cancers demonstrates a correlation between higher SIRT1 expression and worse OS in liver and lung cancers (5). These clinical data are consistent with the experimental ones reported for HCC where SIRT1 activation correlates to malignancy through MALAT1, a lncRNA highly expressed in various cancers including $\operatorname{HCC}(64,68)$.

Furthermore, colorectal and GC do not show the correlation between OS and SIRT1 expression (5). SIRT1 overexpression has been reported either positively associated to FP or negatively associated to OS. Contradictory results also concern SIRT1 IHC and mRNA expression and prognosis of GC, suggesting that this class of lysine-deacetylases is not a reliable biomarker for GC.

In contrast, little evidence is available in the literature concerning the role (if any) of SIRT1 in the pituitary gland. In 
the testis, the role of SIRT1 is reported to be associated with spermatogenesis, fertility, and differentiation, but not testicular cancer (7).

In light of the literature presented here, further basic research is warranted in order to establish possible therapeutic strategies for ovarian, thyroid and pancreatic cancer, where a protumorigenic role for SIRT1 has been established. Particular attention should be paid to the role of miRNAs in regulating SIRT1 levels (miR-212 in thyroid cancer; miR-494, miR-138-5p, miR-601, and miR-217 in PC; miR-204 in HCC). Furthermore, given the growing importance of lncRNAs in cancer pathology, the involvement of MALAT1 should be exploited. For instance, by measuring its levels in HCC biopsies and, possibly, by

\section{REFERENCES}

1. Wu W, Zhang L, Lin J, Huang H, Shi B, Lin X, et al. Hypermethylation of the HIC1 promoter and aberrant expression of HIC1/SIRT1 contribute to the development of thyroid papillary carcinoma. Oncotarget (2016) 7:84416-27. doi: 10.18632/oncotarget.12936

2. Herranz D, Maraver A, Canamero M, Gomez-Lopez G, Inglada-Perez L, Robledo M, et al. SIRT1 promotes thyroid carcinogenesis driven by PTEN deficiency. Oncogene (2013) 32:4052-6. doi: 10.1038/onc.2012.407

3. Klieser E, Urbas R, Stattner S, Primavesi F, Jager T, Dinnewitzer A, et al. Comprehensive immunohistochemical analysis of histone deacetylases in pancreatic neuroendocrine tumors: HDAC5 as a predictor of poor clinical outcome. Hum Pathol. (2017) 65:41-52. doi: 10.1016/j.humpath.2017. 02.009

4. Pinton G, Nilsson S, Moro L. Targeting estrogen receptor beta (ERbeta) for treatment of ovarian cancer: importance of KDM6B and SIRT1 for ERbeta expression and functionality. Oncogenesis (2018) 7:15. doi: 10.1038/s41389-018-0027-9

5. Wang C, Yang W, Dong F, Guo Y, Tan J, Ruan S, et al. The prognostic role of Sirtl expression in solid malignancies: a meta-analysis. Oncotarget (2017) 8:66343-51. doi: 10.18632/oncotarget.18494

6. Das A, Huang GX, Bonkowski MS, Longchamp A, Li C, Schultz MB, et al. Impairment of an Endothelial $\mathrm{NAD}(+)-\mathrm{H} 2 \mathrm{~S}$ signaling network is a reversible cause of vascular aging. Cell (2018) 173:74-89 e20. doi: 10.1016/j.cell.2018.02.008

7. Tatone, C. Di Emidio G, Barbonetti A, Carta G, Luciano AM, Falone S, et al. Sirtuins in gamete biology and reproductive physiology: emerging roles and therapeutic potential in female and male infertility. Hum Reprod Update (2018) 24:267-89. doi: 10.1093/humupd/dmy003

8. Frazzi R, Valli R, Tamagnini I, Casali B, Latruffe N, Merli F. Resveratrolmediated apoptosis of hodgkin lymphoma cells involves SIRT1 inhibition and FOXO3a hyperacetylation. Int $J$ Cancer (2013) 132:1013-21. doi: 10.1002/ijc. 27748

9. Ma CH, Chiua YC, Wu CH, Jou IM, Tu YK, Hung CH, et al. Homocysteine causes dysfunction of chondrocytes and oxidative stress through repression of SIRT1/AMPK pathway: A possible link between hyperhomocysteinemia and osteoarthritis. Redox Biol. (2018) 15:504-12. doi: 10.1016/j.redox.2018. 01.010

10. Sun T, Jiao L, Wang Y, Yu Y, Ming L. SIRT1 induces epithelial-mesenchymal transition by promoting autophagic degradation of E-cadherin in melanoma cells. Cell Death Dis. (2018) 9:136. doi: 10.1038/s41419-017-0167-4

11. Yu EJ, Kim SH, Kim HJ, Heo K, Ou CY, Stallcup MR, et al. Positive regulation of beta-catenin-PROX1 signaling axis by DBC1 in colon cancer progression. Oncogene (2016) 35:3410-8. doi: 10.1038/onc.2015.401

12. Choupani, J. Mansoori Derakhshan S, Bayat S, Alivand MR. Shekari Khaniani, M. Narrower insight to SIRT1 role in cancer: A potential therapeutic target to control epithelial-mesenchymal transition in cancer cells. J Cell Physiol. (2018) 233:4443-57. doi: 10.1002/jcp.26302 monitoring its expression during therapy administration or disease progression.

\section{AUTHOR CONTRIBUTIONS}

RF has been studied SIRT1 in cancer and is currently assessing a possible epigenetic regulation for this enzyme. RF conceived, wrote, and formatted the manuscript.

\section{FUNDING}

This work has been funded by Azienda Unità Sanitaria Locale IRCCS Reggio Emilia, Italy.

13. Lv J, Jiang S, Yang Z, Hu W, Wang Z, Li T, et al. PGC-1alpha sparks the fire of neuroprotection against neurodegenerative disorders. Ageing Res Rev. (2018) 44:8-21. doi: 10.1016/j.arr.2018.03.004

14. Jiang S, Yang Z, Di S, Hu W, Ma Z, Chen F, et al. Novel role of forkhead box O 4 transcription factor in cancer: Bringing out the good or the bad. Semin Cancer Biol. (2018) 50:1-12. doi: 10.1016/j.semcancer.2018.04.007

15. Jiang S, Li T, Yang Z, Hu W, Yang, Y. Deciphering the roles of FOXO1 in human neoplasms. Int J Cancer (2018). doi: 10.1002/ijc.31338

16. Xin Z, Ma Z, Hu W, Jiang S, Yang Z, Li T, et al. FOXO1/3: Potential suppressors of fibrosis. Ageing Res Rev. (2018) 41:42-52. doi: 10.1016/j.arr.2017.11.002

17. Tseng RC, Lee CC, Hsu HS, Tzao C, Wang YC. Distinct HIC1-SIRT1-p53 loop deregulation in lung squamous carcinoma and adenocarcinoma patients. Neoplasia (2009) 11:763-70. doi: 10.1593/neo.09470

18. Calin GA, Croce CM. MicroRNA signatures in human cancers. Nat Rev Cancer (2006) 6:857-66. doi: 10.1038/nrc1997

19. Knight JR, Milner J. SIRT1, metabolism and cancer. Curr Opin Oncol. (2012) 24:68-75. doi: 10.1097/CCO.0b013e32834d813b

20. Henderson JT, Webber EM, Sawaya GF. Screening for ovarian cancer: updated evidence report and systematic review for the us preventive services task force. JAMA (2018) 319:595-606. doi: 10.1001/jama.2017.21421

21. Oliver KE, McGuire WP. Ovarian cancer and antiangiogenic therapy: caveat emptor. J Clin Oncol. (2014) 32:3353-6. doi: 10.1200/JCO.2014.57.4574

22. Kobel M, Kalloger SE, Boyd N, McKinney S, Mehl E, Palmer C, et al. Ovarian carcinoma subtypes are different diseases: implications for biomarker studies. PLoS Med. (2008) 5:e232. doi: 10.1371/journal.pmed.0050232

23. Mvunta DH, Miyamoto T, Asaka R, Yamada Y, Ando H, Higuchi S, et al. Overexpression of SIRT1 is associated with poor outcomes in patients with ovarian carcinoma. Appl Immunohistochem Mol Morphol. (2017) 25:415-21. doi: 10.1097/PAI.0000000000000316

24. Mvunta DH, Miyamoto T, Asaka R, Yamada Y, Ando H, Higuchi S, et al. SIRT1 Regulates the chemoresistance and invasiveness of ovarian carcinoma cells. Transl Oncol. (2017) 10:621-31. doi: 10.1016/j.tranon.2017.05.005

25. Shuang T, Wang M, Zhou Y, Shi C. Over-expression of Sirtl contributes to chemoresistance and indicates poor prognosis in serous epithelial ovarian cancer (EOC). Med Oncol. (2015) 32:260. doi: 10.1007/s12032-015-0706-8

26. Park SH, Cheung LW, Wong AS, Leung PC. Estrogen regulates Snail and Slug in the down-regulation of E-cadherin and induces metastatic potential of ovarian cancer cells through estrogen receptor alpha. Mol Endocrinol. (2008) 22:2085-98. doi: 10.1210/me.2007-0512

27. Akhter MZ, Sharawat SK, Kumar V, Kochat V, Equbal Z, Ramakrishnan $M$, et al. Aggressive serous epithelial ovarian cancer is potentially propagated by EpCAM(+)CD45(+) phenotype. Oncogene (2018) 37:2089103. doi: 10.1038/s41388-017-0106-y

28. Qin J, Liu Y, Lu Y, Liu M, Li M, Li J, et al. Hypoxia-inducible factor 1 alpha promotes cancer stem cells-like properties in human ovarian cancer cells by upregulating SIRT1 expression. Sci Rep. (2017) 7:10592. doi: 10.1038/s41598-017-09244-8 
29. Kitahara CM, Sosa JA. The changing incidence of thyroid cancer. Nat Rev Endocrinol (2016) 12:646-653. doi: 10.1038/nrendo. 2016.110

30. Dang CV, O’Donnell KA, Zeller KI, Nguyen T, Osthus RC, Li F. The c-Myc target gene network. Semin Cancer Biol. (2006) 16:253-64. doi: 10.1016/j.semcancer.2006.07.014

31. Menssen A, Hydbring P, Kapelle K, Vervoorts J, Diebold J, Luscher B, et al. The c-MYC oncoprotein, the NAMPT enzyme, the SIRT1-inhibitor DBC1, and the SIRT1 deacetylase form a positive feedback loop. Proc Natl Acad Sci USA. (2012) 109:E187-96. doi: 10.1073/pnas.1105304109

32. Yuan J, Minter-Dykhouse K, Lou Z. A c-Myc-SIRT1 feedback loop regulates cell growth and transformation. J Cell Biol. (2009) 185:203-11. doi: $10.1083 /$ jcb. 200809167

33. Han HS, Choi BH, Kim JS, Kang G, Koo SH. Hepatic Crtc2 controls whole body energy metabolism via a miR-34a-Fgf21 axis. Nat Commun/ (2017) 8:1878. doi: 10.1038/s41467-017-01878-6

34. Qiang L, Sample A, Liu H, Wu X, He YY. Epidermal SIRT1 regulates inflammation, cell migration, and wound healing. Sci Rep. (2017) 7:14110. doi: 10.1038/s41598-017-14371-3

35. Frazzi R, Tigano, M. The multiple mechanisms of cell death triggered by resveratrol in lymphoma and leukemia. Int J Mol Sci. (2014) 15:4977-93. doi: 10.3390/ijms15034977

36. Duntas, L. H. Resveratrol and its impact on aging and thyroid function. $J$ Endocrinol Invest. (2011) 34:788-92.

37. Shih A, Davis FB, Lin HY, Davis PJ. Resveratrol induces apoptosis in thyroid cancer cell lines via a MAPK- and p53-dependent mechanism. J Clin Endocrinol Metab. (2002) 87:1223-32. doi: 10.1210/jcem.87. 3.8345

38. Li D, Bai L, Wang T, Xie Q, Chen M, Fu Y, Wen Q. Function of miR-212 as a tumor suppressor in thyroid cancer by targeting SIRT1. Oncol Rep. (2018) 39:695-702. doi: 10.1007/s12094-017-1772-5

39. Notta F, Hahn SA, Real FX. A genetic roadmap of pancreatic cancer: still evolving. Gut (2017) 66:2170-78. doi: 10.1136/gutjnl-2016313317

40. Gurusamy KS, Riviere D, C.van Laarhoven JH, Besselink M, Abu-Hilal $\mathrm{M}$, Davidson BR, et al. Cost-effectiveness of laparoscopic versus open distal pancreatectomy for pancreatic cancer. PLoS ONE (2017) 12:e0189631. doi: 10.1371/journal.pone.0189631

41. Liu Y, Li X, Zhu S, Zhang JG, Yang M, Qin Q, et al. Ectopic expression of miR-494 inhibited the proliferation, invasion and chemoresistance of pancreatic cancer by regulating SIRT1 and c-Myc. Gene Ther. (2015) 22:72938. doi: $10.1038 /$ gt.2015.39

42. Tian S, Guo X, Yu C, Sun C, Jiang J. miR-138-5p suppresses autophagy in pancreatic cancer by targeting SIRT1. Oncotarget (2017) 8:11071-82. doi: 10.18632/oncotarget.14360

43. Cao $\mathrm{W}$, Jin $\mathrm{H}$, Zhang $\mathrm{L}$, Chen $\mathrm{X}$, Qian $\mathrm{H}$. Identification of miR601 as a novel regulator in the development of pancreatic cancer. Biochem Biophys Res Commun. (2017) 483:638-44. doi: 10.1016/j.bbrc.2016. 12.090

44. Deng S, Zhu S, Wang B, Li X, Liu Y, Qin Q, et al. Chronic pancreatitis and pancreatic cancer demonstrate active epithelial-mesenchymal transition profile, regulated by miR-217-SIRT1 pathway. Cancer Lett. (2014) 355:184-91. doi: 10.1016/j.canlet.2014.08.007

45. Oon CE, Strell C, Yeong KY, Ostman A, Prakash J. SIRT1 inhibition in pancreatic cancer models: contrasting effects in vitro and in vivo. Eur J Pharmacol. (2015) 757:59-67. doi: 10.1016/j.ejphar.2015. 03.064

46. Bolm L, Kasmann L, Paysen A, Karapetis C, Rades D, Wellner UF, et al. Multimodal Anti-tumor approaches combined with immunotherapy to overcome tumor resistance in esophageal and gastric cancer. Anticancer Res. (2018) 38:3231-42. doi: 10.21873/anticanres. 12588

47. Wang K, Liu R, Li J, Mao J, Lei Y, Wu J, et al. Quercetin induces protective autophagy in gastric cancer cells: involvement of Akt-mTORand hypoxia-induced factor lalpha-mediated signaling. Autophagy (2011) 7:966-78. doi: 10.4161/auto.7.9.15863

48. Humbert M, Medova M, Aebersold DM, Blaukat A, Bladt F, Fey MF, et al. Protective autophagy is involved in resistance towards MET inhibitors in human gastric adenocarcinoma cells. Biochem Biophys Res Commun. (2013) 431:264-9. doi: 10.1016/j.bbrc.2012.12.120

49. Hu X, Sui X, Li L, Huang X, Rong R, Su X, et al. Protocadherin 17 acts as a tumour suppressor inducing tumour cell apoptosis and autophagy, and is frequently methylated in gastric and colorectal cancers. J Pathol. (2013) 229:62-73. doi: 10.1002/path.4093

50. Lee IH, Cao L, Mostoslavsky R, Lombard DB, Liu J, Bruns NE, et al. A role for the NAD-dependent deacetylase Sirt1 in the regulation of autophagy. Proc Natl Acad Sci USA. (2008) 105:3374-9. doi: 10.1073/pnas.07121 45105

51. Fullgrabe J, Klionsky DJ, Joseph, B. Histone post-translational modifications regulate autophagy flux and outcome. Autophagy (2013) 9:1621-3. doi: 10.4161 /auto. 25803

52. Qiu G, Li X, Che X, Wei C, He S, Lu J, et al. SIRT1 is a regulator of autophagy: Implications in gastric cancer progression and treatment. FEBS Lett. (2015) 589:2034-42. doi: 10.1016/j.febslet.2015.05.042

53. Yang $\mathrm{Q}$, Wang $\mathrm{B}$, Gao $\mathrm{W}$, Huang $\mathrm{S}$, Liu Z, Li W, et al. SIRT1 is downregulated in gastric cancer and leads to G1-phase arrest via NF-kappaB/Cyclin D1 signaling. Mol Cancer Res. (2013) 11:1497-507. doi: 10.1158/1541-7786.MCR-13-0214

54. Wu S, Jiang J, Liu J, Wang X, Gan Y, Tang Y. Meta-analysis of SIRT1 expression as a prognostic marker for overall survival in gastrointestinal cancer. Oncotarget (2017) 8:62589-99. doi: 10.18632/oncotarget. 19880

55. Shen $\mathrm{X}, \mathrm{Li} \mathrm{P}, \mathrm{Xu} \mathrm{Y}$, Chen $\mathrm{X}$, Sun $\mathrm{H}$, Zhao $\mathrm{Y}$, et al. Association of sirtuins with clinicopathological parameters and overall survival in gastric cancer. Oncotarget (2017) 8:74359-70. doi: 10.18632/oncotarget. 20799

56. Noguchi A, Kikuchi K, Zheng H, Takahashi H, Miyagi Y, Aoki I, et al. SIRT1 expression is associated with a poor prognosis, whereas DBC1 is associated with favorable outcomes in gastric cancer. Cancer Med. (2014) 3:1553-61. doi: $10.1002 / \mathrm{cam} 4.310$

57. Brooks CL, Gu W. How does SIRT1 affect metabolism, senescence and cancer?. Nat Rev Cancer (2009) 9:123-8. doi: 10.1038/nrc2562

58. Song NY, Surh YJ. Janus-faced role of SIRT1 in tumorigenesis. Ann N Y Acad Sci. (2012) 1271:10-9. doi: 10.1111/j.1749-6632.2012. 06762.x

59. Marti N, Bouchoucha N, Sauter KS, Fluck CE. Resveratrol inhibits androgen production of human adrenocortical H295R cells by lowering CYP17 and CYP21 expression and activities. PLoS ONE (2017) 12:e0174224. doi: 10.1371/journal.pone.0174224

60. Shibata T, Arai Y, Totoki Y. Molecular genomic landscapes of hepatobiliary cancer. Cancer Sci. (2018) 109:1282-91. doi: 10.1111/cas. 13582

61. Broutier L, Mastrogiovanni G, Verstegen MM, Francies HE, Gavarro LM, Bradshaw CR, et al. Human primary liver cancer-derived organoid cultures for disease modeling and drug screening. Nat Med. (2017) 23:1424-35. doi: $10.1038 / \mathrm{nm} .4438$

62. Wang S, Wan T, Ye M, Qiu Y, Pei L, Jiang R, et al. Nicotinamide riboside attenuates alcohol induced liver injuries via activation of SirT1/PGC1alpha/mitochondrial biosynthesis pathway. Redox Biol. (2018) 17:89-98. doi: 10.1016/j.redox.2018.04.006

63. Zhou X, Chen J, Chen L, Feng X, Liu Z, Zeng ZH, et al. Negative regulation of Sirtuin 1 by AMP-activated protein kinase promotes metformin-induced senescence in hepatocellular carcinoma xenografts. Cancer Lett. (2017) 411:111. doi: $10.1016 /$ j.canlet.2017.09.027

64. Hou Z, Xu X, Zhou L, Fu X, Tao S, Zhou J, et al. The long non-coding RNA MALAT1 promotes the migration and invasion of hepatocellular carcinoma by sponging miR-204 and releasing SIRT1. Tumour Biol. (2017) 39:1010428317718135. doi: 10.1177/10104283177 18135

65. Yoshimoto R, Mayeda A, Yoshida M, Nakagawa S. MALAT1 long non-coding RNA in cancer. Biochim Biophys Acta (2016) 1859:192-9. doi: 10.1016/j.bbagrm.2015.09.012

66. Fang D, Yang H, Lin J, Teng Y, Jiang Y, Chen J, et al. 17beta-estradio regulates cell proliferation, colony formation, migration, invasion and promotes apoptosis by upregulating miR-9 and thus degrades 
MALAT-1 in osteosarcoma cell MG-63 in an estrogen receptorindependent manner. Biochem Biophys Res Commun. (2015) 457:500-6. doi: 10.1016/j.bbrc.2014.12.114

67. Michalik KM, You X, Manavski Y, Doddaballapur A, Zornig $\mathrm{M}$, et al. Long noncoding RNA MALAT1 regulates endothelial cell function and vessel growth. Circ Res. (2014) 114:1389-97. doi: 10.1161/CIRCRESAHA.114.303265

68. Lin R, Maeda S, Liu C, Karin M, Edgington TS. A large noncoding RNA is a marker for murine hepatocellular carcinomas and a spectrum of human carcinomas. Oncogene (2007) 26:851-8. doi: 10.1038/sj.onc.1209846
Conflict of Interest Statement: The author declares that the research was conducted in the absence of any commercial or financial relationships that could be construed as a potential conflict of interest.

Copyright (C) 2018 Frazzi. This is an open-access article distributed under the terms of the Creative Commons Attribution License (CC BY). The use, distribution or reproduction in other forums is permitted, provided the original author(s) and the copyright owner(s) are credited and that the original publication in this journal is cited, in accordance with accepted academic practice. No use, distribution or reproduction is permitted which does not comply with these terms. 